\title{
Three cases report of idiopathic angiosarcoma of the head and neck and literature review
}

\author{
Hong-Zhi Jiang, Xian-Hua Jin, Xin-Yi Huang, Tian-Tian Dong, Hong-Jing Zhang, Xiao-Han Liu, \\ Jian-Xin Xia \\ Department of Dermatology, the Second Hospital of Jilin University, Changchun, China \\ Correspondence to: Jian-Xin Xia. Department of Dermatology, the Second Hospital of Jilin University, Changchun 130041, China. \\ Email: xiajx@jlu.edu.cn.
}

\begin{abstract}
Herein, we reported 3 cases of angiosarcoma (AS) of the head and neck in old patients, and the etiology, pathogenesis, histopathology, immunohistochemistry, diagnosis, differential diagnosis and prognosis were discussion. Case 1, a male patient aged 86 years old was admitted due to purplish erythema on the head and face and progressive purplish edema in the orbit of the eye for 3 years. He was misdiagnosed with connective tissue disease in another hospital, and a second biopsy and histopathological examination confirmed the AS of the head and neck. Case 2, a male patient aged 85 years were admitted due to erythemalike hyperplasia in the left anterior head with necrosis and scar formation at the center for 2 months. He was misdiagnosed with folliculitis in another hospital, and histopathological examination confirmed the AS of the head and neck. Case 3, a male patient aged 87 years were admitted due to large erythema in the right scalp with ulcer and scar formation for 1 month, and histopathological examination confirmed the AS of the head and neck. One of them was lost to follow, one died 5 months after confirmed diagnosis, and one achieved favorable outcome after radiotherapy and received further follow up. The early clinical manifestations of the three patients were different and lacked characteristics. The disease progressed rapidly and the prognosis was poor. At present, surgery combined with postoperative radiotherapy is the preferred treatment. Through this article, the following conclusions can be obtained: erythema in the head and face of the elderly, rapid progresses and painful, pathological examinations must be performed, and changes in blood vessels and vascular endothelial cells with or without crack formation and red blood cell extravasation should be carefully observed during pathological examinations in case of misdiagnosis. It is hoped that these three cases can serve as a reminder for clinicians, for reducing misdiagnosis and miss diagnosis, choosing appropriate treatment methods, and judging their prognosis. Therefore, early diagnosis and surgical treatment are extremely important to improve the prognosis of AS patients.
\end{abstract}

Keywords: Angiosarcoma (AS); head and neck; radiotherapy; case report

Submitted Oct 19, 2019. Accepted for publication Apr 10, 2020.

doi: 10.21037/apm-19-397

View this article at: http://dx.doi.org/10.21037/apm-19-397

\section{Introduction}

Angiosarcoma (AS) is a malignant vascular tumor, accounting for about $2 \%$ of all soft tissue sarcomas. It can occur in any part of the body, but $60 \%$ of AS is found in the skin or superficial soft tissues. Clinically, it is divided into idiopathic AS of the head and neck, post-radiation AS, and lymphedema-related AS. Herein, we reported 3 case of AS of the head and neck in old patients whose early clinical manifestations were different and lacked characteristics. Initially, the skin lesions are often atypical, and two of them were misdiagnosed in other hospital. Therefore, being familiar with the clinical features of idiopathic AS of the head and neck is crucial for the early diagnosis and early treatment, which is important for the prolongation of survival time. 

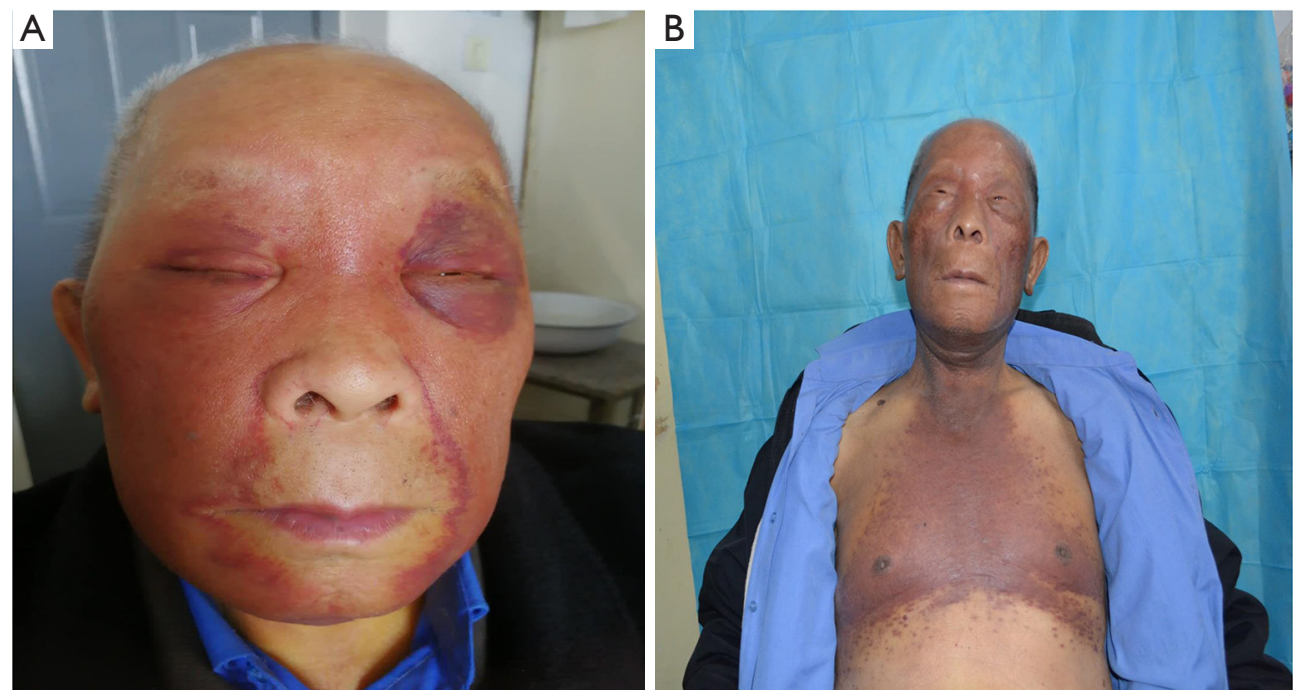

Figure 1 Clinical manifestations of case 1. (A) Lesions on the head and face; (B) radiation therapy for 5 months.

\section{Case presentation}

\section{Case 1}

A male patient aged 86 years was admitted due to purplish erythema on the head and face and progressive purplish edema in the orbit of the eye for 3 years. The patient developed erythema of unknown cause with mild swelling but free of itching on the left eye in April 2016, and no treatment was administered. The erythema gradually enlarged, and involved the right eyelid and bilateral cheeks without itching. In the local hospital, biopsy and subsequent pathological examination indicated "connective tissue disease" in May 2017. Then, oral hydroxychloroquine (FenLe) was administered (2 capsules, twice daily), but the skin lesions remained, the erythema became dark and purple, further expanded, and soon involved the majority of the cheeks and the neck, bleeding and purpura were found at the edge of skin lesions, and the left eye edema became more evident. Then, he visited our hospital for further diagnosis and treatment in October 2018.

Physical examination: The general condition was favorable, the blood pressure (BP) was $150 / 80 \mathrm{mmHg}$, detection of serum RPR + TPPA and HIV showed negative. The patient has a history of hypertension for 20 years which is well controlled by oral medications. He has a history of coronary heart disease for 10 years. He denies a history of diabetes. He denies history of chronic diseases such as heart diseases and cerebrovascular diseases, family history, and mental illness. He has no history of infectious diseases such as hepatitis and tuberculosis. He denies trauma histories. He denies histories of food allergies and reports allergies to tape and penicillin. He had never had tests for related genes. The systematic physical examination showed no abnormality.

Dermatological examination: a large, soft skin lesion with diffuse irregular purple erythema was found on the face and neck. A large purple-like lesion was noted in the left eyelid with evident (Figure 1).

Histopathological examination: spindle-shaped cells infiltrated the dermal collagen fibers and divided these fibers; fissure-like region was observed without evident atypia, but red blood cell exosmosis was noted around the fissure-like region (Figure 2).

Immunohistochemistry: the spindle-shaped cells were positive for CD31 (Figure 3A), CD34 (Figure 3B), and D2-40 (Figure 3C). Then, idiopathic AS of the head and neck was diagnosed. This patient received radiotherapy in our hospital for 5 months. In November 2018, nine radiotherapy sessions were performed from the target dose DT of 6 to $50 \mathrm{~Gy} / 3 \mathrm{f}$. The adverse reactions were mild. Low white blood cells and low platelets appeared during the radiotherapy. Symptomatic and supportive treatments were effective. When the second radiotherapy was performed in April 2019, chest metastases appeared. The target area was adjusted, and radiation therapy was continued. The target area dose DT began from 14 to $50 \mathrm{~Gy} / 3 \mathrm{f}$. Five radiotherapy sessions were performed. The adverse reactions were mild, including low white blood cells and low platelets. 
Symptomatic and supportive treatment were effective. Dark red pigmentation remains in the anterior chest skin lesions after radiotherapy. Currently, the general condition of this patient was favorable (Figure 1B). He was receiving follow up (Figure 4).

\section{Case 2}

A male patient aged 85 years were admitted due to an erythema or lump on the left forehead for 4 months and necrosis and ulcer at the center of the lesion for about 2 months. He developed erythema and pimples on the left forehead of unknown cause in February 2015, which progressively enlarged to form a mass. The lesion

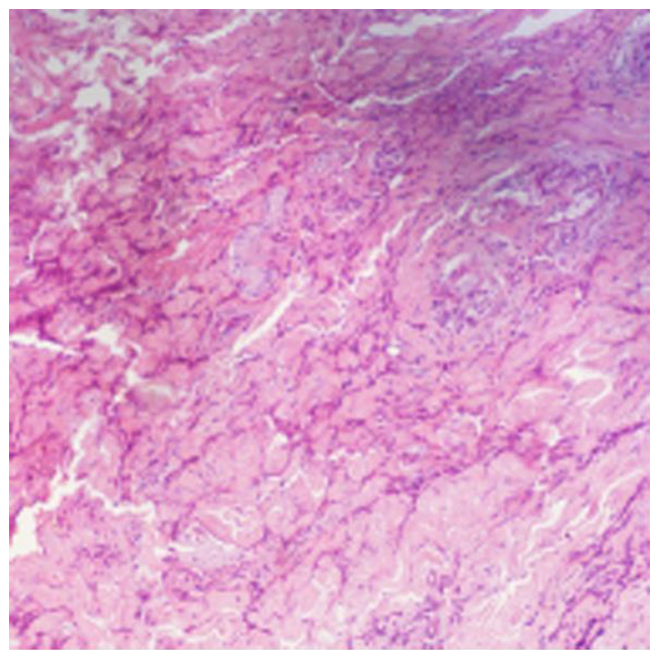

Figure 2 HE staining of case $1(\times 100)$. became ulcerated without itching in April 2015. He was diagnosed with folliculitis in a local hospital, and then oral cephalosporins and antibiotic ointment were administered for the treatment of folliculitis. However, the skin lesion remained unchanged, further enlarged and became ulcerated at the center. Therefore, he visited out hospital for further treatment in June 2015.

Physical examination: the general condition was favorable. He had a history of hypertension for 10 years, and currently received treatment with oral pressurelowering drugs with favorable blood pressure control. He denies history of chronic diseases, family history, and mental illness. He had never had tests for related genes. Dermatological examination: a mass sized $3 \mathrm{~cm} \times 5 \mathrm{~cm}$ was found on the left forehead, with an ulcer at the center and a sense of infiltration on palpation (Figure 5).

Histopathological examination: The acanth layer was slightly thickened, the dermal vessels and lymphatics were dilated and hyperemic, and diffuse infiltration of tumor cells was observed in the whole dermis layer. Tumor cells were slightly larger than lymphocytes, and lumen- and fissurelike structures formed in some regions, with obvious atypia. Lymphocyte infiltration was observed around tumor cells (Figure 6). Immunohistochemistry: tumor cells were positive to CD31 (Figure 7A), CD34 (Figure 7B) and D240 (Figure $7 C$ ), but negative to $\mathrm{S} 100$, and then facial AS was diagnosed. After the diagnosis was confirmed, the patient was transferred to a local hospital for radiotherapy and he was lost to follow-up (Figure 8).

Case 3

A male patient aged 87 years was admitted due to a large
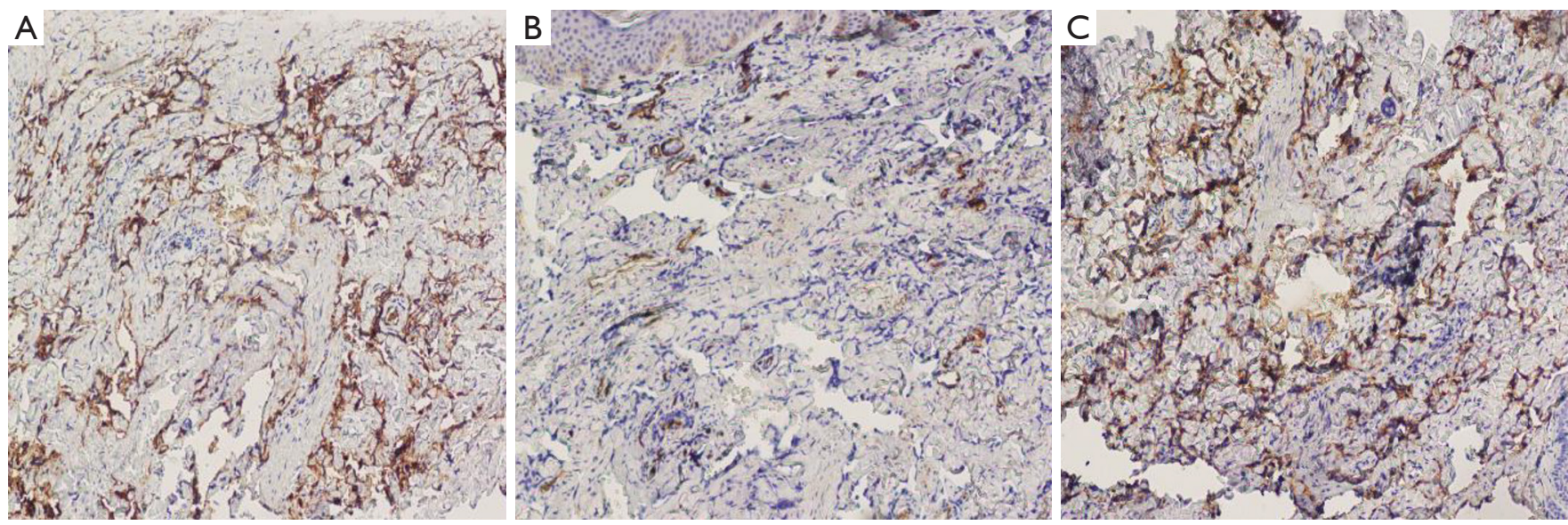

Figure 3 Immunohistochemistry of case 1 (SP, ×100). (A) CD31 (+) (SP×100); (B) CD34 (+) (SP×100); (C) D2-40 (+) (SP, ×100). 


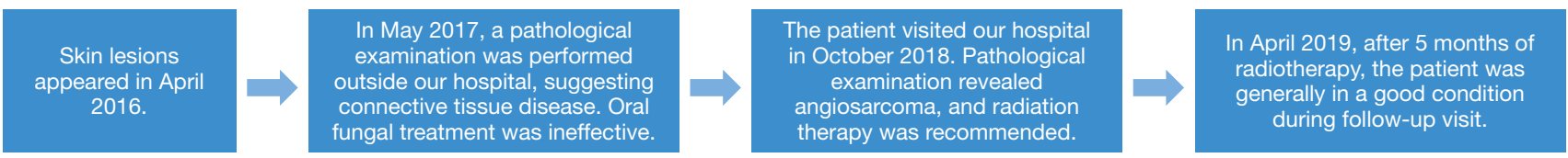

Figure 4 Timeline picture of the case 1 .

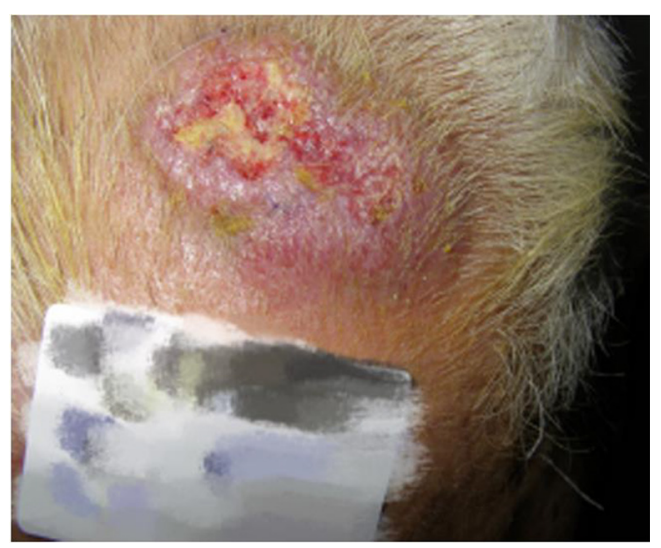

Figure 5 Lesions on the head and face of case 2.

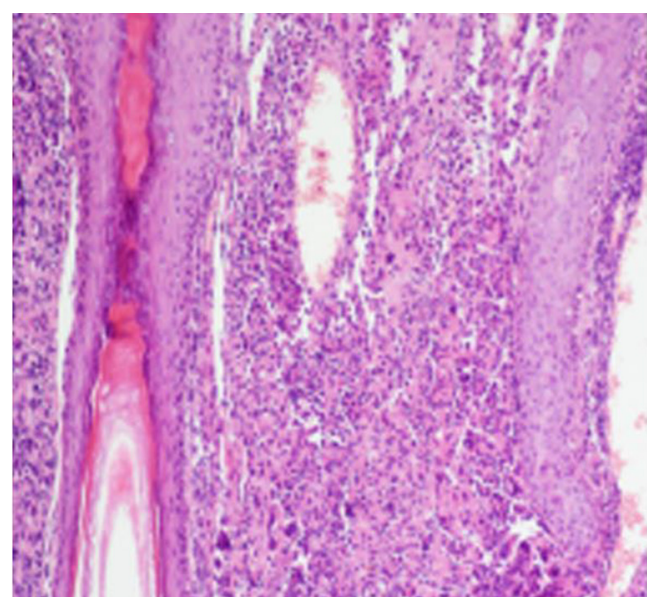

Figure $6 \mathrm{HE}$ staining of case $2(\times 100)$.

purple erythema on the right scalp for 5 months and ulcer and scar formation at the skin lesion for 1 month. In March 2016, he had a trauma on the right scalp, and developed erythema and swelling without itching. The erythema enlarged gradually and the swelling became evident. In June 2016, the skin lesion became ulcerated and bleeding with purulent exudation, scarring and pain. Then, he visited our hospital for further diagnosis and treatment in July 2016.

He denies history of chronic diseases, family history, and mental illness. He had never had tests for related genes. Physical examination: the general condition was good, and systemic examination failed to show abnormalities.

Dermatological examination: a purple plaque sized $13 \mathrm{~cm} \times 15 \mathrm{~cm}$ was found on the right scalp, with massive ulcer, scarring and purulent exudation (Figure 9).

Histopathological examination: fissures formed between the dermal collagen fibers, and the tumor cells with large, dark nucleus infiltrated the margins of the fissures (Figure 10).

Immunohistochemistry: atypical cells were positive to CD31 (Figure 11A) and CD34 (Figure 11B), and Ki67 positive cells accounted for 30\% (Figure 11C). AS of the head was diagnosed. The patient returned to a local hospital for further treatment, but he died in January 2017 (Figure 12).

Consent was obtained from relatives of the patient for publication of this report and any accompanying images.

\section{Discussion}

AS is divided into idiopathic AS of the head and neck, post-radiation AS, and lymphedema-associated AS and accounts for only $2 \%$ of soft tissue sarcomas. The ratio of male to female is about 2:1 in AS patients, and it has a poor prognosis. The idiopathic AS of the head and neck is more common in the elderly, and the clinical manifestations of AS of the head and neck include erythema, papules, blisters, purpura, nodules and masses. The highly malignant AS may develop ulcer and bleeding. In addition, local recurrence and early metastasis are also common in patients with AS of the head and neck (1).

The etiology and pathogenesis of AS are still unknown, and might be related to the trauma, radiotherapy, ionizing radiation, chemical exposure and chronic lymphedema. Of the 3 patients in this report, 1 had a history of trauma before the occurrence of as. Reed et al. (2) reported 6 cases of AS on the head, of whom 5 had a history of local trauma, and 1 had severe trauma. This supports the theory of traumatic origin, but there is no definitive evidence to support the Causal relationship between trauma and cutaneous AS. A 

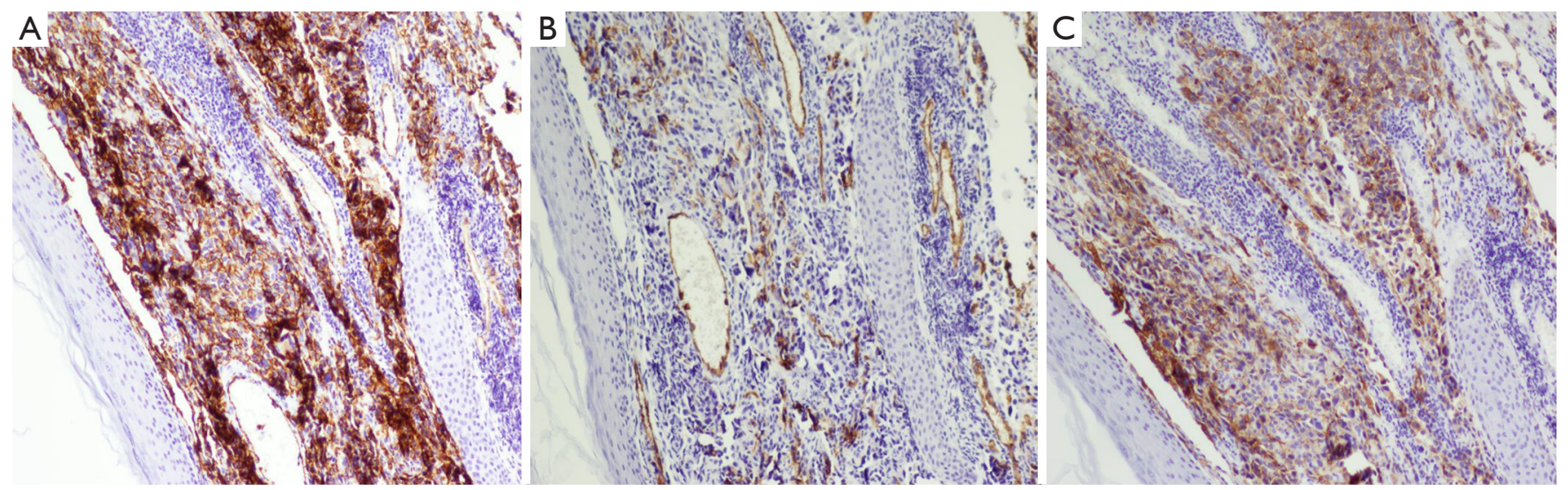

Figure 7 Immunohistochemistry of case 2 (SP, ×100). (A) CD31(+) (SP, $\times 100)$; (B) CD34(+) (SP, $\times 100)$; (C) D2-40 (+) (SP, $\times 100)$.

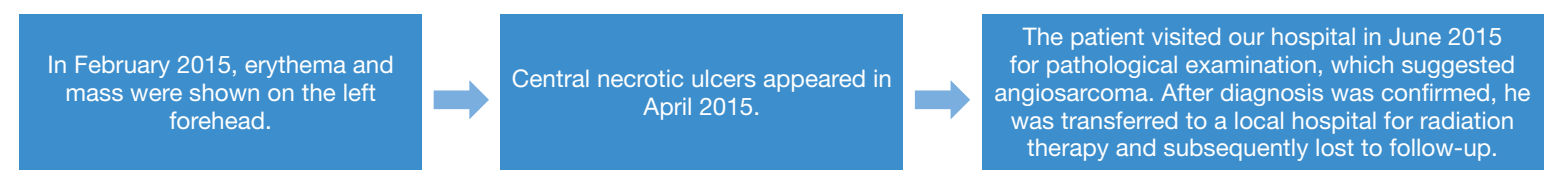

Figure 8 Timeline picture of the case 2.

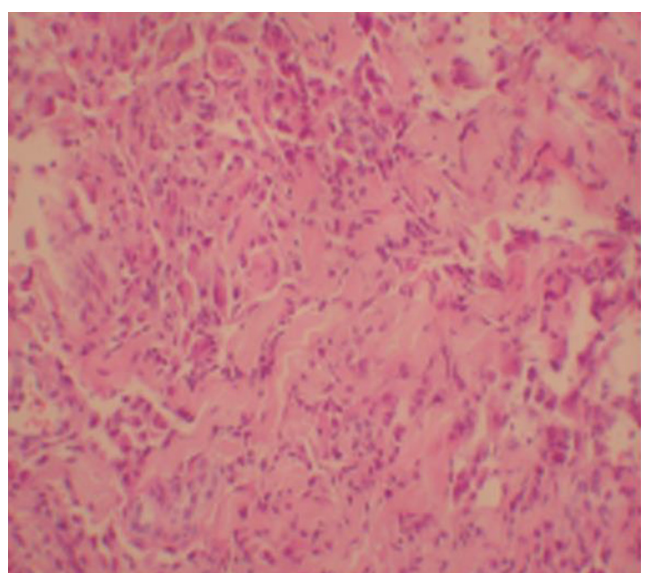

Figure 9 Lesions on the head and face of case 3.

variety of factors may be involved in the pathogenesis and progression of AS, and vascular endothelial growth factor (VEGF) is related to the regulation of endothelial cell proliferation, angiogenesis, and vascular permeability. Amo et al. (3) investigated the serum VEGF-D in 11 patients with AS of the head and 18 healthy controls. Compared with the control group, the serum VEGF-D significantly increased in patients with AS, and it further increased with progression of AS. At the same time, these investigators detected serum angiopoietin-2 and they found the serum

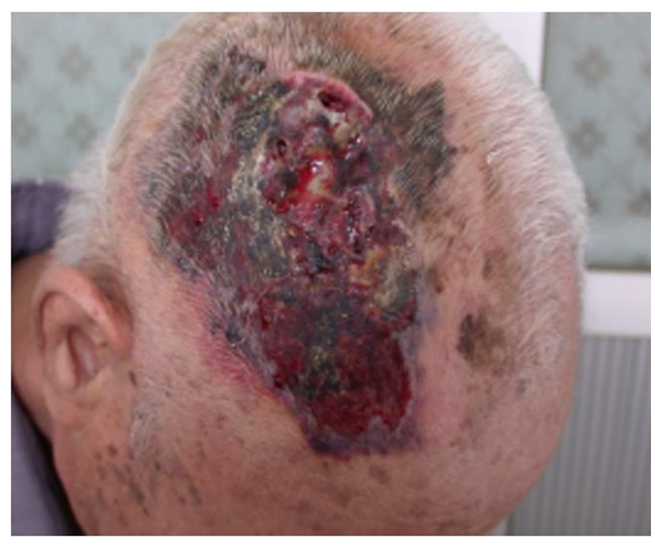

Figure $10 \mathrm{HE}$ staining of case $3(\times 200)$.

angiopoietin-2 also significantly increased in patients with AS as the disease progressed. The loss of vascular endothelial cadherin may be associated with decreased cell adhesion, which is an important factor associated with elevated invasiveness of AS (4).

Histopathological examination showed that the tumor was located in the dermis, the boundary was unclear, and it consisted of numerous interweaving blood vessels of varying sizes and displayed invasive growth. Endothelial cells encapsulate the collagen bundle, showing the 

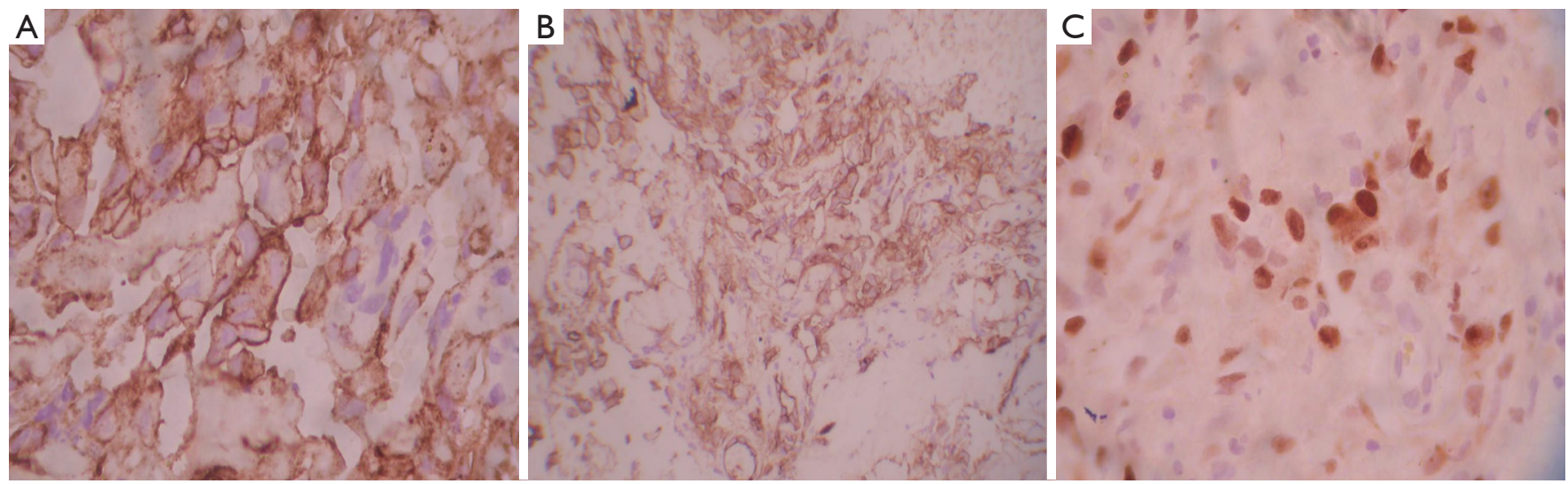

Figure 11 Immunohistochemistry of case 3 (SP, ×200). (A) CD34(+) (SP, ×200); (B) CD31(+) (SP, ×200); (C) Ki-67 30\% (SP, ×200).
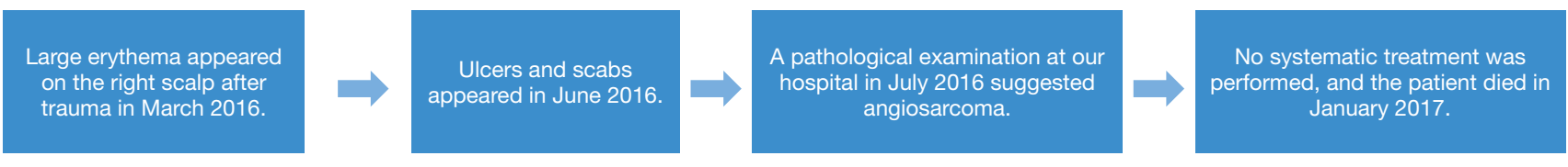

Figure 12 Timeline picture of the case 3.

dissecting pattern. Only one layer of endothelial cells was seen in the lumen. It is easy to misdiagnoze the tumor when there is no evident atypia. In the well to moderately differentiated tumor, multiple layers of atypical endothelial cells can be found on the wall of the blood vessel, and intracavity proliferation is often observed (5). In the poorly differentiated tumor, the tumor presents with sarcomatoid with spindle-shaped cells or solid tumor, and the overflow of red blood cells is rare, but the appendage and blood vessels are often destroyed (6). In this report, fissure-like cavities with red blood cell extravasation were the dominant features on histopathological examination in case 1; the solid tumor-like change was the dominant feature in case 2; the collagen-fiber fissure-like cavities were the dominant feature in case 3 . Immunohistochemistry plays an important role in the diagnosis of AS, and CD31, CD34 and FVIII are the common markers of vascular endothelial cells. Especially, CD31 is a specific marker of vascular endothelial cells. D240 is a lymphatic endothelial marker. In the study of Cuda et al. (7), the positive rate of D2-40 in AS was about $60 \%$. In a study of Kahn et al. (8), AS was subdivided into two groups originating from different progenitor cell types: one is limited to the differentiation along the vascular endothelium lineage and the other along the lymphoid and vascular lineage. Therefore, D2-40 expression can be found in the AS.
The early clinical manifestations of these 3 patients were different in this report: 2 were misdiagnosed; case 1 showed facial purple erythema, case 2 displayed an ulcer on the head, and case 3 showed ecchymosis on the head. In case 1 , only dark purple erythema occurred initially around the eye, and pathological examination displayed mucoid deposition in the superficial dermis, suggesting connective tissue disease. In case 2, this patient was misdiagnosed with folliculitis. Therefore, once the erythema on the head and face shows rapid progression and pain, pathological examination is necessary for confirmed diagnosis. On pathological examination, the blood vessels and vascular endothelial cells with or without fissure formation and extravasation of red blood cells should be carefully checked, aiming to avoid misdiagnosis. In addition, AS of the head and neck should be differentiated from the basal cell carcinoma, squamous cell carcinoma, connective tissue disease, deep mycosis, cellulitis, sarcoidosis, hemangioma and metastatic carcinoma of the skin.

The preferred treatment for AS is still surgical resection and postoperative radiotherapy. In case 1 , surgery is infeasible because skin lesion on the face and neck is very large and therefore, radiation therapy was employed. Although the skin lesion was improved on the head, large ecchymoses appeared on the front chest. Currently, he 
received radiotherapy with the front chest as a target. It has been reported that paclitaxel can be used to treat advanced AS. Penel et al. (9) performed a phase II clinical trial in 30 patients with unresectable or metastatic AS, and evaluated the efficacy of paclitaxel. Fujisawa et al. (10) did a retrospective study and found that the prognosis of paclitaxel combined with radiotherapy is better than that of conventional surgery combined with radiotherapy. Paclitaxel combined with radiotherapy should be superior to radiotherapy alone. Due to limited conditions, case 1 failed to receive both paclitaxel chemotherapy and radiotherapy at the same time. Their results supported the use of paclitaxel as first-line therapy for advanced AS.

The prognosis of AS is extremely poor, and most patients with AS often die 2-3 years after diagnosis, with a median survival time of 15-24 months and a 5-year survival rate of $7.1-33.0 \%$ (11). In case 1 , only radiotherapy was employed, the skin lesion on the face and neck was improved, but skin lesion occurred. The food intake, sleep and physical performance were acceptable in this patient, he was receiving follow up and he had survived for 3 years and 8 months. Therefore, the radiotherapy is preferred for patients who are not the candidates for surgery. In case 3 , the skin lesion on the scalp was severe and surgery was infeasible. After the diagnosis, he only received analgesic treatment and died 5 months later. A recent meta-analysis (12) reported that the adverse prognostic factors in patients with cAS include being older than 70 years, tumors larger than $5 \mathrm{~cm}$ in diameter and AS on the head. In the present report, these factors were found in 3 patients, suggesting a poor prognosis. Recent studies have found that high expression of CD98 in the tumor cells predicts a poor prognosis (13). Tumor size is also an important predictor of survival. Naka et al. (14) reported the prognosis of patients with skin lesion $\leq 5 \mathrm{~cm}$ was significantly better than that of patients with skin lesion $>5 \mathrm{~cm}(\mathrm{P}<0.01)$, which was further confirmed by univariate and multivariate analyses.

The limitations of this case report are that there are few cases, leading to no effective statistical analysis conducted. Besides, both the early skin lesions and the follow-ups of the two patients were missed.

\section{Conclusions}

Therefore, early diagnosis and surgical treatment are extremely important to improve the prognosis of AS patients.

\section{Acknowledgments}

Funding: None.

\section{Footnote}

Conflicts of Interest: All authors have completed the ICMJE uniform disclosure form (available at http://dx.doi. org/10.21037/apm-19-397). The authors have no conflicts of interest to declare.

Ethical Statement: The authors are accountable for all aspects of the work in ensuring that questions related to the accuracy or integrity of any part of the work are appropriately investigated and resolved. All procedures performed in studies involving human participants were in accordance with the Declaration of Helsinki (as revised in 2013). Consent was obtained from relatives of the patient for publication of this report and any accompanying images.

Open Access Statement: This is an Open Access article distributed in accordance with the Creative Commons Attribution-NonCommercial-NoDerivs 4.0 International License (CC BY-NC-ND 4.0), which permits the noncommercial replication and distribution of the article with the strict proviso that no changes or edits are made and the original work is properly cited (including links to both the formal publication through the relevant DOI and the license). See: https://creativecommons.org/licenses/by-nc-nd/4.0/.

\section{References}

1. Mark RJ, Poen JC, Tran LM, et al. Angiosarcoma. A report of 67 patients and a review of the literature. Cancer 1996;77:2400-6.

2. Reed RJ, Palomeque FE, Hairston MA, 3rd, et al. Lymphangiosarcomas of the scalp. Arch Dermatol 1966;94:396-402.

3. Amo Y, Masuzawa M, Hamada Y, et al. Serum concentrations of vascular endothelial growth factor-D in angiosarcoma patients. Br J Dermatol 2004;150:160-1.

4. Tanioka M, Ikoma A, Morita K, et al. Angiosarcoma of the scalp: absence of vascular endothelial cadherin in primary and metastatic lesions. Br J Dermatol 2001;144:380-3.

5. Mendenhall WM, Mendenhall CM, Werning JW, et al. Cutaneous angiosarcoma. Am J Clin Oncol 2006;29:524-8.

6. Chen LQ, Zeng XS, Jiang WQ, et al. Cutaneous angiosarcoma on face and scalp of the aged: clinical 
and pathological study of nine cases. J Clin Dermatol 2005;34:722-5.

7. Cuda J, Mirzamani N, Kantipudi R, et al. Diagnostic utility of Fli-1 and D2-40 in distinguishing atypical fibroxanthoma from angiosarcoma. Am J Dermatopathol 2013;35:316-8.

8. Kahn HJ, Bailey D, Marks A. Monoclonal antibody D240, a new marker of lymphatic endothelium, reacts with Kaposi's sarcoma and a subset of angiosarcomas. Mod Pathol 2002;15:434-40.

9. Penel N, Bui BN, Bay JO, et al. Phase II trial of weekly paclitaxel for unresectable angiosarcoma: the ANGIOTAX Study. J Clin Oncol 2008;26:5269-74.

10. Fujisawa Y, Yoshino K, Kadono T, et al.

Chemoradiotherapy with taxane is superior to conventional surgery and radiotherapy in the management of cutaneous angiosarcoma: a multicentre, retrospective study. Br J Dermatol 2014;171:1493-500.

11. Holden CA, Spittle MF, Jones EW. Angiosarcoma of the face and scalp, prognosis and treatment. Cancer 1987;59:1046-57.

12. Shin JY, Roh SG, Lee NH, et al. Predisposing factors for poor prognosis of angiosarcoma of the scalp and face: Systematic review and meta-analysis. Head Neck 2017;39:380-6.

13. Shimizu A, Kaira K, Okubo Y, et al. Prognostic impact of LAT1 and CD98 expression in cutaneous angiosarcoma. Neoplasma 2017;64:283-8.

14. Naka N, Ohsawa M, Tomita Y, et al. Angiosarcoma in Japan. A review of 99 cases. Cancer 1995;75:989-96.

Cite this article as: Jiang HZ, Jin XH, Huang XY, Dong TT, Zhang HJ, Liu XH, Xia JX. Three cases report of idiopathic angiosarcoma of the head and neck and literature review. Ann Palliat Med 2020;9(4):2333-2340. doi: 10.21037/apm-19-397 